\title{
Hydrodynamic Performance of a Vortex Generator
}

\author{
P.A. Brandner ${ }^{1}$ and G.J. Walker ${ }^{2}$ \\ ${ }^{1}$ Faculty of Maritime Transport and Engineering \\ Australian Maritime College, Launceston Tasmania, 7248 AUSTRALIA \\ ${ }^{2}$ School of Engineering \\ University of Tasmania, Hobart, Tasmania, 7001 AUSTRALIA
}

\begin{abstract}
The performance of a vortex generator intended for a marine application is investigated experimentally in a cavitation tunnel. Several tests were made as a preliminary study to gain basic knowledge of an initial design concept. Investigations included flow visualisation of local flow and wake trajectory, cavitation inception and occurrence, and measurement of boundary layer profiles both upstream and downstream of the vortex generator. Boundary layers were measured in the non-cavitating condition only. Two upstream boundary layer profiles were tested and were found to have little influence on cavitation inception for a range of incidence.
\end{abstract}

\section{Introduction}

Vortex generators (VGs) have been used successfully in many aeronautical applications for reduction of separation in both internal and external flows. The vortices (or turbulence) created transfer low energy fluid from the surface into the mainstream, and bring higher energy fluid from the mainstream down to the surface where the higher kinetic energy level is able to withstand a greater pressure rise before separation occurs. Hoerner [1] provides a discussion and review of their use for lifting surfaces such as wings and control surfaces. Anderson [2,3] details computational investigations into the use of VGs in an " $\mathrm{S}$ " shaped aero-engine inlet duct for minimisation of flow distortion at the engine face. In this instance their use is not strictly to control separation but to reduce secondary flows that produce non-uniform flow at the engine face.

The present work is motivated by the use of VGs in a marine application to control viscous flows (both separation and pump face distortion) within a waterjet propulsor inlet duct. Flow problems within inlet ducts of waterjet propulsors have been investigated by the present authors [4] and the use of VGs is seen as a promising means of improving a range of flow problems. This method also has the advantage of being easy to retrofit to existing vessels.

Aspects that need to be considered include the profile, size, position, sign and magnitude of incidence, cavitation effects and structural requirements. In aeronautical applications a range of geometries have been tried but the ones in most common use tend to be simple vane types. Odelal [5] provides a review of a range of different VG geometries and results of cavitation tunnel tests for application to hydraulic and marine equipment. Odelal concludes that more investigation is required before recommendations can be made on which type may be more useful in particular applications.

For the present investigation a delta-shaped VG was chosen for practical reasons, including structural requirements, the need to avoid fouling with debris and a belief that this type may produce less drag. An experimental series of tests has been made as a preliminary investigation to gain a basic understanding of flow behaviour and indicate where improvements might be made. The tests included flow visualisation, the influence of boundary layer (BL) thickness, and cavitation inception and occurrence.

\section{Experimental Overview}

\section{Vortex Generator}

A delta-shaped VG was chosen for the practical considerations mentioned above. The height/base length ratio was suggested by results of an aeronautical optimisation study. The model scale is $1: 2$ based on a full scale VG height of $75 \mathrm{~mm}$ that could be used in a typical waterjet inlet duct for a modern $100 \mathrm{~m}$ long fast ferry. The model geometry is shown in Figure 1. The base plate and fin are a practical design feature enabling retro fitting to the inlet duct wall, and may be simply cut from "T" section stock. The model VG was fixed to a rotatable acrylic penetration such that the centreline of the trailing edge remained fixed. The penetration was machined with a protractor scale to allow setting of the incidence.

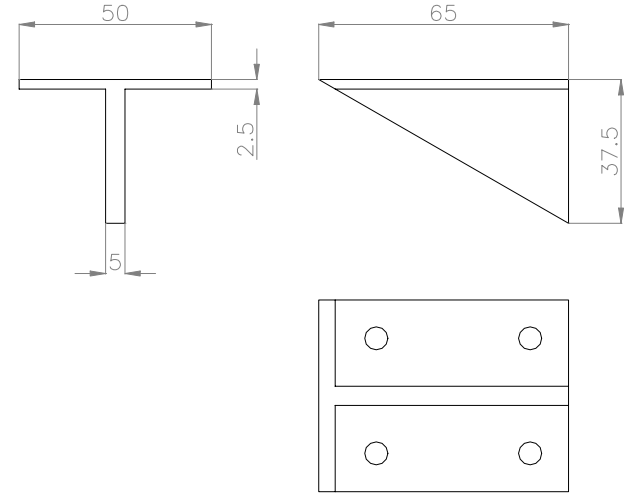

Figure 1. Model VG Geometry (Dimensions in mm).

\section{Cavitation Tunnel Experimental Setup}

All tests where performed in the Tom Fink Cavitation Tunnel, a closed recirculating variable pressure water tunnel. The test section dimensions are $0.6 \mathrm{~m} \times 0.6 \mathrm{~m}$ cross section $\times 2.6 \mathrm{~m}$ long. The velocity may be varied from 2 to $12 \mathrm{~m} / \mathrm{s}$ and the centreline static pressure from 4 to $400 \mathrm{kPa}$ absolute. Typical capabilities include the testing of propellers, foils, waterjets and other such devices. Studies may involve the investigation of steady and unsteady flows, two-phase flows including cavitation, turbulence and hydro-acoustics. Full details of the tunnel and its capabilities are given in Brandner and Walker [4].

The test section was set up with the VG located on the ceiling $1900 \mathrm{~mm}$ from the test section entrance. A BL thickener can be attached to the ceiling $300 \mathrm{~mm}$ from the test section entrance. At each of these positions, and a third $500 \mathrm{~mm}$ downstream of the $\mathrm{VG}$, access for a probe was available for measuring BL profiles.

Parameters measured during testing include tunnel pressure, velocity, temperature and dissolved oxygen content. Online instrumentation is used for automatic control of tunnel pressure and velocity as well as real time data monitoring and acquisition. 
The test section pressure is measured using 2 Rosemount Model 3051C Smart absolute pressure transducers in parallel. Test section velocity is derived from the contraction differential measured using 2 Rosemount Model 1151 Smart differential pressure transducers in parallel. One of each pressure transducer pair has a lower range to improve measurement precision at lower pressures and velocities respectively.

Water temperature is measured using a Rosemount Model 244 temperature transducer. Dissolved Oxygen content is measured using a Rosemount Model 499 Dissolved Oxygen sensor. Pitot tube pressures (as well as tunnel instrument pressures) were measured sequentially using a Validyne Model DP15TL differential pressure transducer via a Model 48J7-1 Scanivalve pressure multiplexer.

Measurement of BL profiles were made using a $1 / 8$ " $(3.13 \mathrm{~mm})$ diameter Pitot-static tube and traverse manufactured by United Sensor Corporation.

\section{Experimental Procedure}

The following tests were performed:

- Measurement of natural/thickened BL profiles;

- Measurement of BL profiles downstream of VG;

- Flow visualisation of local/wake flow using air injection;

- Observation of cavitation inception and occurrence.

BL profiles were measured at three longitudinal positions along the centreline of the test section ceiling: at the position where a BL thickener can be fitted; at the position of the VG; and at a position $500 \mathrm{~mm}$ downstream of the VG. Natural BL profiles were measured at each of these positions and thickened profiles at the location of the $\mathrm{VG}$ and at the downstream position. Profiles were also measured with the VG fitted at the downstream position to investigate the mixing effect - in noncavitating conditions only. Profiles were typically measured at intervals of $1 \mathrm{~mm}$ up to $20 \mathrm{~mm}$ from the wall, $2 \mathrm{~mm}$ up to $30 \mathrm{~mm}$, $5 \mathrm{~mm}$ up to $50 \mathrm{~mm}$ and $10 \mathrm{~mm}$ thereafter.

With the VG fitted, downstream profiles were measured at VG incidence angles of $10,20,30$ and $40^{\circ}$. As the measured velocities would not be parallel with the Pitot static tube head for these tests yaw sensitivity measurements were made. These showed errors of less than $3 \%$ in the dynamic pressure for up to $10^{\circ}$ probe yaw.

A range of devices for BL thickening in the cavitation tunnel has been tested. Conventional arrays of rods or spires and screens have generally been unsuccessful due to vibration, acoustic resonance, cavitation, and poor velocity and turbulence profiles. The device used for the present investigation is a spanwise fence with a serrated top. The most successful geometry trialled consists of a fence with contiguous triangular elements of base $20 \mathrm{~mm}$ and height $10 \mathrm{~mm}$. The drag of the triangular "teeth" introduces the momentum deficit needed to thicken the BL, and the flow around the teeth introduces streamwise vortices of a scale comparable to the tooth width. The vortices are very effective in promoting transverse mixing and rapidly distributing the momentum deficit over the BL height. A realistic turbulence distribution is also achieved with this method. The BL height is adjusted by raking the fence backward and generators with angles varying from 30 to $90^{\circ}$ have been used. For these tests a $30^{\circ}$ angle was used. As the teeth are generally well within the natural BL the inception cavitation number for this type of generator is significantly lower than for other devices.

All BL measurements were made at $8 \mathrm{~m} / \mathrm{s}$ test section velocity. Data from all transducers was acquired at $300 \mathrm{~Hz}$ and averaged over 10 seconds. Acquired data were corrected for small temporal changes using the contraction differential during post processing.

Flow visualisation was by air injection via a reverse Pitot tube located $600 \mathrm{~mm}$ upstream of the VG inserted $20 \mathrm{~mm}$ into the flow. Provided the bubbles are sufficiently small in diameter they are effective for flow visualisation or flow tracking. A procedure for calculating relative bubble velocities based on bubble size, length scales and velocity is given by Brennan [6]. Small bubbles can be produced using a reverse Pitot tube provided its internal diameter is small (in this case approximately $0.3 \mathrm{~mm}$ ) and there is some turbulence present to promote break up of larger bubbles and minimise coalescence. The bubbles were illuminated using a $150 \mathrm{~W}$ flood lamp and images recorded using a conventional digital camera. Flow visualisation was performed at each angle of incidence $\left(10,20,30\right.$ and $\left.40^{\circ}\right)$ at $5 \mathrm{~m} / \mathrm{s}$ test section velocity. This was found to be compatible with the lighting and camera used.

Cavitation inception and occurrence was investigated at each angle of incidence $\left(10,20,30\right.$ and $\left.40^{\circ}\right)$. The results provide general information including the nature of the cavitation, its type, noise level, possibility of causing destruction, etc. From the inception and the variation of the type of cavitation with the Cavitation number, a so-called "cavitation bucket" may be plotted describing overall behaviour. All cavitation testing was done at $8 \mathrm{~m} / \mathrm{s}$ test section velocity and the dissolved oxygen content was maintained at approximately $30 \%$ of saturation at atmospheric pressure. The cavitation number used for the present testing is defined as;

$$
\sigma=\frac{P_{\infty}-P_{V}}{1 / 2 \rho V^{2}}
$$

where $P_{\infty}$ is the static pressure at the test section centreline, $P_{V}$ the water vapour pressure, $V$ the freestream velocity and $\rho$ the fluid density. The variation of Cavitation Number was achieved by changing the static pressure while maintaining a fixed velocity. Cavitation images were recorded using a conventional $35 \mathrm{~mm}$ SLR camera and synchronised stroboscopic lighting.

\section{Results}

\section{Boundary Layer Profiles}

Figure 2 shows the natural and thickened BL profiles at the three positions of interest along the test section ceiling: where the boundary thickener may be fitted; where the VG is fitted; and where velocity profiles are measured downstream of the VG. The natural BL at $300 \mathrm{~mm}$ from entrance is approximately $12 \mathrm{~mm}$ thick, growing to $30 \mathrm{~mm}$ at 1900 and $37 \mathrm{~mm}$ at $2400 \mathrm{~mm}$. In each case the power law index is approximately 6.5 .

For the thickened BL at the position of the VG and at the downstream position, approximate values for the thickness and power law index are $62 \mathrm{~mm}$ and 9.5 respectively. The natural BL at the thickener position is shown for comparison.

\section{Downstream Boundary Layer Profiles}

Figure 3 shows the influence of the $\mathrm{VG}$ on the BL $500 \mathrm{~mm}$ downstream on the test section centreline. Due to deflection of the wake these measurements provide only part of the information on the effects of the VG. However they provide a basis for future more detailed surveys. Flow visualisation shows that the probe remained within the wake plume and that the wake trajectory is deflected less that one VG chord length from the centreline in all cases. Notwithstanding the limited nature of the results it can be seen from the results that the VG performs well at 10 and $20^{\circ}$ incidence. For both natural and thickened BLs the 
mixing effect extended to approximately $65 \mathrm{~mm}$ from the wall for all angles of incidence.
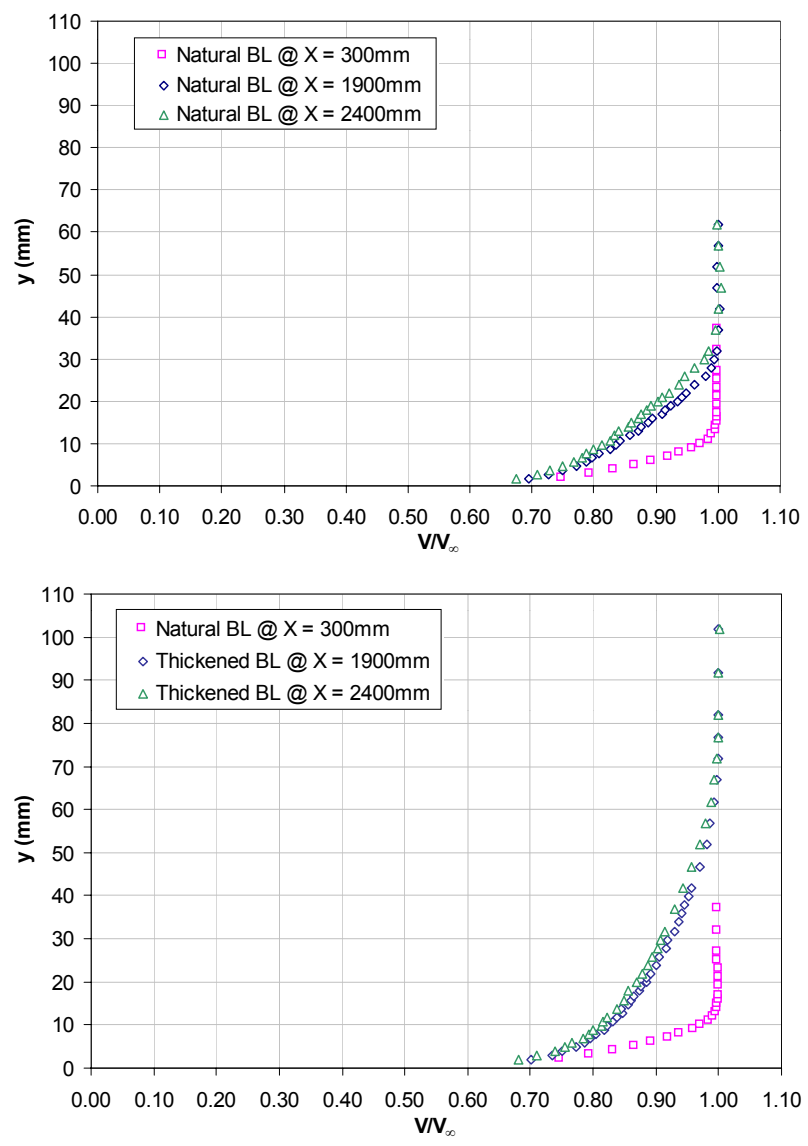

Figure 2. Natural and Thickened BL Profiles at Three Locations along the Test Section: the BL Thickener; the VG: 500mm Downstream of the VG.
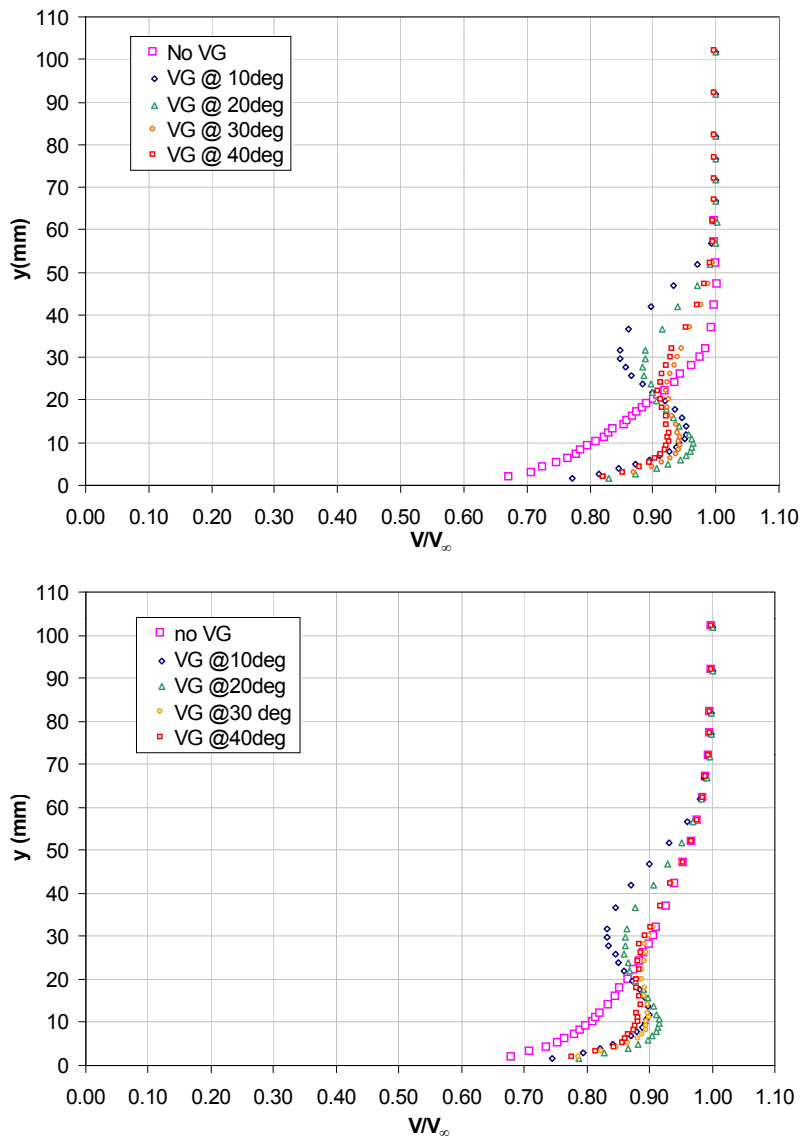

Figure 3. The Effect of the VG on the Natural and Thickened BLs $500 \mathrm{~mm}$ Downstream of the VG at 10, 20,30 and $40^{\circ}$ Incidence.

\section{Flow Visualisation}

Flow visualisation revealed the nature of the flow and the wake trajectory at each angle of incidence. Figure 4 shows the VG at $10^{\circ}$ incidence. The vortex core can be seen as well as the extent and deflection of the wake plume. At $20^{\circ}$ incidence the vortex generated undergoes so called "vortex bursting" (or stall for a delta wing) as shown in Figure 5. Katz and Plotkin [7] provide a discussion of this phenomenon and a review of delta wing performance. The vortex burst may be advantageous for mixing although it is a likely source of increased drag. At $30^{\circ}$ incidence the vortex bursting occurs closer to the leading edge and at $40^{\circ}$ incidence there is induced separation along the entire leading edge.

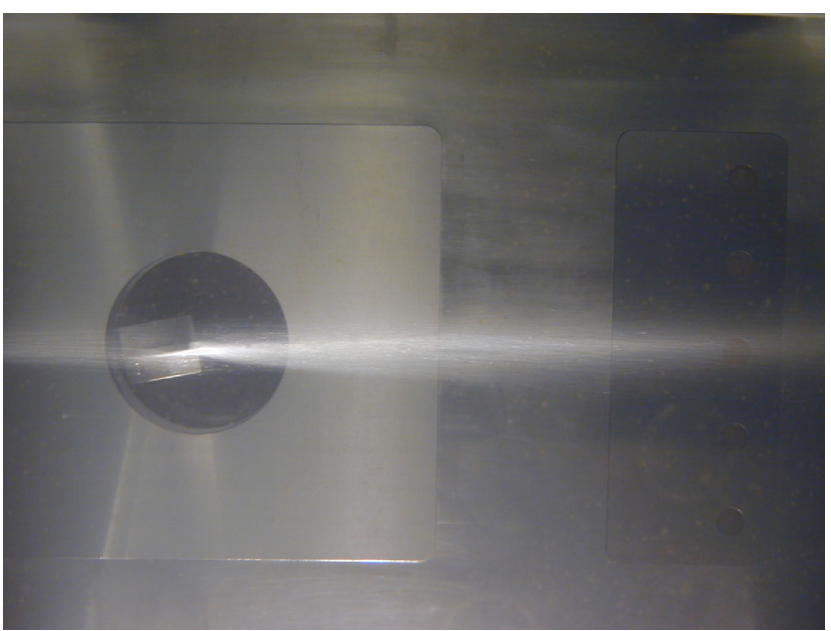

Figure 4. Flow Visualisation of wake at $10^{\circ}$ Incidence.

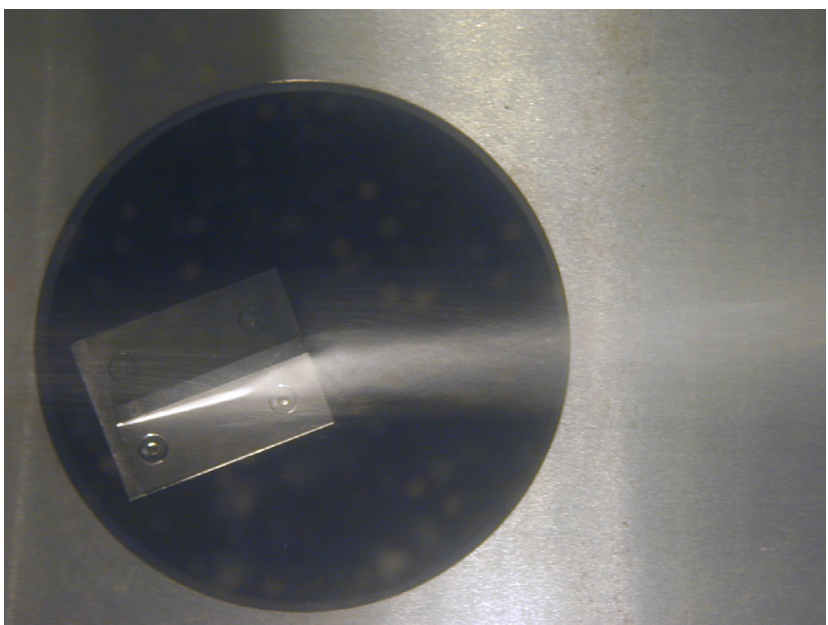

Figure 5. Flow Visualisation of Vortex Bursting at $20^{\circ}$ Incidence.

\section{Cavitation Inception and Occurrence}

At 10,20 and $30^{\circ}$ incidence (where a vortex was formed) cavitation inception occurred at the very leading edge of the VG where the vortex is initiated. Inception was intermittent and was defined in this case as occurring when the small vortex cavity appeared for $50 \%$ of the time. At $10^{\circ}$ incidence the vortex cavity would quickly grow with decreasing cavitation number until it extended to the trailing edge. With further decrease in $\sigma$ a sheet cavity formed on the leading edge and grew in extent from the start of the leading edge until the trailing edge was reached. After this there was a breakdown of stable flow and an unsteady sheet cavity forms with cloud cavitation downstream of the VG. A similar transition to unstable flow occurred at 20 and $30^{\circ}$ incidences, except that the initial vortex cavity did not grow beyond the vortex burst. A sequence of photographs for a range of $\sigma$ values is shown in Figure 6 for the thickened BL. The vortex burst is easily seen. 


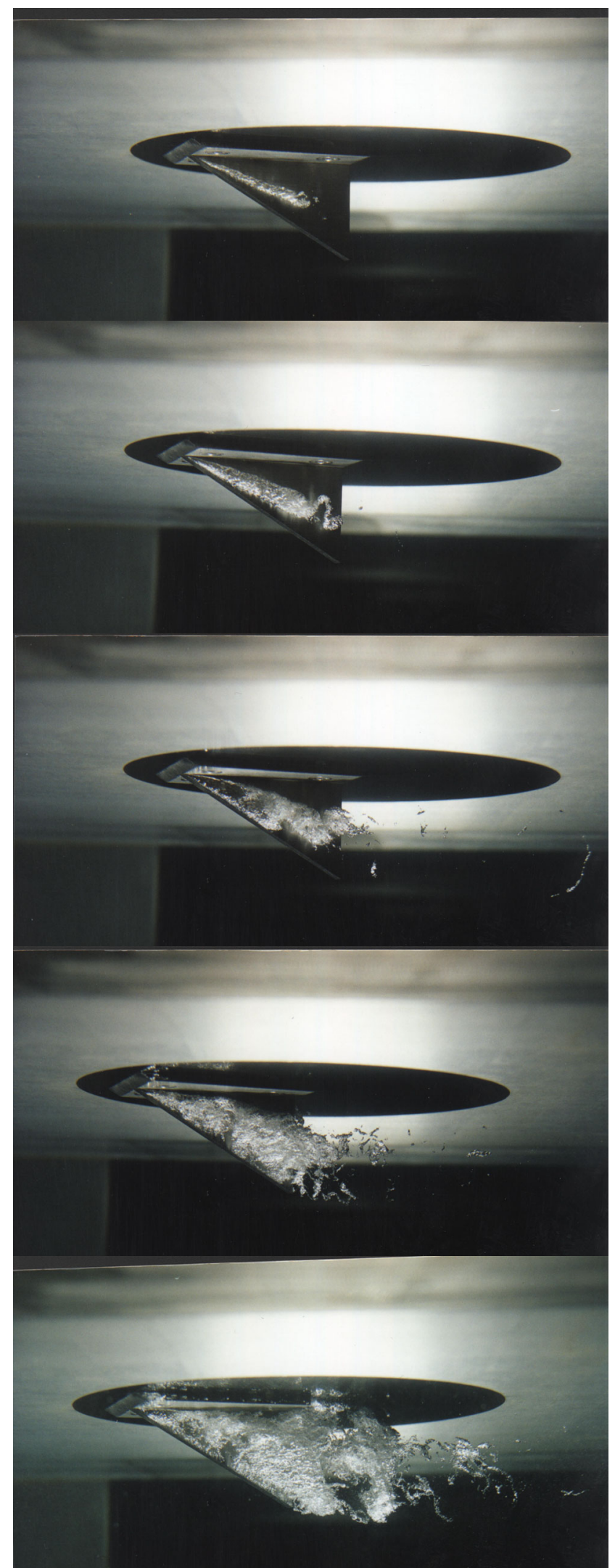

Figure 6. Cavitation Occurrence at $20^{\circ}$ Incidence with a Thickened BL at Cavitation Numbers of 2.0, 1.5, 1.0, 0.75 and 0.5.

While no noise measurements were made, the vortex cavitation at the higher $\sigma$ values was noted as significantly more noisy than that from the leading edge sheet and cloud cavitation. At $40^{\circ}$ incidence, where the flow is fully separated along the leading edge, there was no change in cavitation type, inception led immediately to unstable sheet or cloud cavitation similar to that for the smaller angles of incidence. In this case inception occured in the vortices of the shear flows resulting from the leading edge separation.

Figure 7 shows the cavitation bucket with lines for inception and vortex and full leading edge cavities. Of interest is the virtually identical results obtained for the natural and thickened BL cases. For inception the results are essentially identical and hence only one cavitation bucket is presented. The almost identical inception $\sigma$ values can be attributed to the similar velocities adjacent to the wall in the natural and thickened boundary cases. These affect the initiation of the vortex and hence cavitation inception.

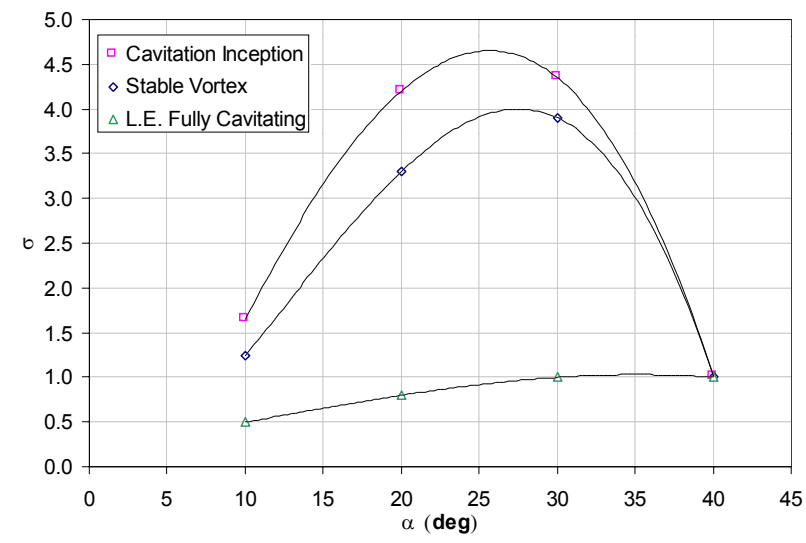

Figure 7. Cavitation Bucket for Both Natural and Thickened BLs.

\section{Conclusions}

A preliminary investigation into the performance of a deltashaped VG for a marine application has been completed. The VG behaves similar to a delta wing in that it undergoes vortex bursting which was evident from flow visualisation and cavitation occurrence. Cavitation inception occurs either at the initiation of the vortex if the flow is attached or within the shear flows of the separated region. For attached flow decreasing $\sigma$ values produce leading edge sheet cavitation which subsequently becomes unstable and causes rapid breakdown of vortex flow. Future studies would include generation of BL profiles closer to a separation situation, leading edge modifications for improved vortex flows and increased inception $\sigma$ values, effects of vortex bursting and effects of the VG aspect ratio.

\section{References}

[1]Hoerner, S.F., Fluid-Dynamic Lift, Hoerner Fluid Dynamics, 1985.

[2]Anderson, B.H., Huang, P.S., Paschal, W.A. and Cavatorta, E., A study on Vortex Control of Inlet Distortion in the ReEngined 727-100 Center Inlet Duct Using Computational Fluid Dynamics , AIAA $30^{\text {th }}$ Aerospace Sciences Meeting and Exhibit., 1992.

[3]Anderson, B.H. and Gibb, J., Application of Computational Fluid Dynamics to the Study of Vortex Control for the Management of Inlet Distortion, AIAA/ASME/SAE $28^{\text {th }}$ Joint Propulsion Conference and Exhibit, 1992.

[4]Brandner, P.A. and Walker G.J., A Waterjet Test Loop for the Tom Fink Cavitation Tunnel, International Conference on Waterjet Propulsion III, Royal Institution of Naval Architects, 2001, 54-57.

[5]Oledal, M., Application of Vortex Generators in Ship Propulsion System Design, ODRA 97' $2^{\text {nd }}$ International Conference on Marine Technology, Computational Mechanics Publications, 1997, 247-255.

[6]Brennan, C.E., Cavitation and Bubble Dynamics, Oxford University Press, 1995.

[7]Katz, J. and Plotkin, A., Low Speed Aerodynamics: From Wing Theory to Panel Methods, McGraw-Hill, 1991. 